\title{
Aproximación a la génesis de la contribución de la densidad en la noción de "ciudad compacta"
}

\author{
José Ramón Navarro Vera \\ Armando Ortuño Padilla \\ Departamento de Geografía Humana. Área de Urbanística y Ordenación del \\ Territorio. Universidad de Alicante.
}

RESUMEN | Como introducción al artículo se traza la génesis del concepto de ciudad compacta a partir de las diferentes variables que lo configuran, explicando cómo éstas se han ido incorporando a esa noción tan extendida en el urbanismo contemporáneo. A continuación se expone un trabajo de investigación desarrollado en el territorio periurbano de Alicante, en el que se relaciona densidad residencial con variables de movilidad. Finalmente, se proponen líneas de investigación que se abren a la luz de la problemática actual de las formas de crecimiento y desarrollo de las ciudades.

PALABRAS CLAVE | Áreas metropolitanas, movilidad, ordenación territorial, desarrollo sostenible.

ABSTRACT $\mid$ As an introduction to the article, the conceptual origins of a compact city will be outlined considering the different variables involved. How these variables have been incorporated into such a widespread concept in the field of contemporary urbanism will be explained. Secondly, a research project developed in the outlying urban areas of Alicante which links residential density with variables of mobility will be presented. Finally, new research lines will be proposed to shed light on the current problems posed by the forms of growth and development of cities.

KEY WORDS | Metropolitan areas, mobility, spatial planning, sustainable development. 
"A medida que va disminuyendo la superficie urbana que toca por habitante, se va aumentando la mortalidad de la población". Ildefonso Cerdá. Teoría General de la Urbanización. Año $1876^{1}$.

En los últimos años, el debate sobre la forma urbana y metropolitana, tanto desde una visión morfológica como cuantitativa del desarrollo espacial, se ha visto alentado y renovado por la extensión del paradigma ambiental ${ }^{2}$. Actualmente, la noción de ciudad compacta como remisión a una cierta idea de la ciudad mediterránea tradicional, densa y con un alto grado de mix funcional, se ha convertido tanto en un paradigma disciplinar vinculado a las estrategias de sostenibilidad ambiental urbana como en un icono verbal del discurso de algunos políticos locales.

En nuestra opinión, la noción de ciudad compacta se corresponde, en general, más con una imagen interpretativa de una realidad espacial que con un modelo generalizado elaborado a partir de los datos de aquella realidad. Se pueden observar dos tendencias: la primera, ideológica, que utiliza la noción de ciudad compacta para legitimar propuestas desde el punto de vista de la sostenibilidad, o como componente, en la misma línea ambiental, de un cierto discurso urbanístico, en ocasiones carente de rigor o incluso contradictorio; y la segunda, derivada de un análisis de la ciudad difusa, en donde ésta aparece como un negativo de la ciudad compacta, y donde la densidad residencial figura como una variable relevante. En un informe publicado por la Unión Europea titulado Urban sprawl in Europe se define así el fenómeno de la dispersión de las actividades residenciales y económicas en el territorio contemporáneo:

"Dispersión urbana es el término utilizado genéricamente para definir físicamente la expansión de las áreas urbanas. La Agencia Europea de Medio Ambiente describe la dispersión como el patrón físico de expansión de baja densidad que se produce en grandes áreas urbanas bajo condiciones de mercado sobre el suelo agrícola circundante. La dispersión es el fenómeno dominante en los límites del espacio de crecimiento urbano e implica escaso control del mismo. Es un desarrollo desigual, disperso y con tendencia a la discontinuidad. El territorio disperso es lo contrario de la ciudad compacta, dominado por los espacios vacíos que indican la ineficiencia de un crecimiento incontrolado"3.

1 La densidad del casco de Barcelona a mediados del siglo XIX, según Cerdá, era de 859 hab/ha, y la media de los barrios oscilaba entre 378 hab/ha y 1.761 hab/ha. Según Baumeister, la densidad más alta en una ciudad europea era la del barrio judío de Praga: 1.428 hab/ha (Soria, 1979). Jane Jacobs (1971) sostiene que las innovaciones en las técnicas urbanas surgieron impulsadas por los problemas derivados del crecimiento de las ciudades. Desde esa posición se puede afirmar que la urbanística moderna no habría surgido si no hubieran existido grandes ciudades en el siglo XIX con sus graves problemas asociados, por eso esta autora mantiene que en la actualidad "limitar el tamaño de las grandes ciudades, como se intenta a veces, a causa de que los más graves problemas son consecuencia de sus dimensiones es reaccionario en extremo".

2 Podemos considerar a Kevin Lynch y Lloyd Rodwin (1958) como los introductores de la noción de forma urbana, en la que la densidad es uno de sus componentes para determinar la relación ente forma y objetivos humanos (buman objectives).

3 Urban sprawl in Europe. The ignored challenge. EEA Report, $n^{\circ}$. 10/2006. En este informe se muestra cómo los territorios con mayor impacto del sprawl en Europa son las regiones con alta población, densidad y actividad económica (Holanda, sur y oeste de Alemania, norte de Italia, región de París) y/o rápido crecimiento económico (Irlanda, Portugal, este de Alemania, región de Madrid). Se resalta que los procesos de desarrollos territoriales de baja densidad son particularmente evidentes en las regiones que se han visto beneficiadas por las ayudas comunitarias. Asimismo, se llama la atención sobre la ocupación del suelo en la costa de España, donde la superficie artificial cubre del orden del $50 \%$ del total del área. 
Desde la protohistoria del urbanismo, la densidad de población fue uno de los indicadores de la calidad de vida urbana. Foucault sostenía que fueron los médicos los primeros especialistas del espacio construido, al plantear la densidad de población, junto al emplazamiento, desplazamientos, y condiciones del hábitat público y privado, como los cuatro problemas fundamentales que había que cuidar para asegurar unas condiciones de vida saludables en las ciudades (Bentham y Foucault, 1979). La cita que encabeza este artículo es un buen ejemplo de ello y de la influencia que el paradigma higiénico ha tenido en los orígenes del urbanismo moderno.

Entre mediados del siglo XIX y del XX, las diferentes propuestas enmarcadas en los tres paradigmas más influyentes en la teoría urbanística -el higiénico, el racional y el "usoniano" - tienen a la densidad residencial como uno de los indicadores diferenciadores. En el CIAM de Bruselas de 1930, Le Corbusier presenta su proyecto de Ville Radieuse donde sitúa torres de $150 \mathrm{~m}$ de altura con densidades de 1.000hab/ha, mientras Gropius, en el mismo encuentro, desarrollaba sus conocidas propuestas de distribución óptima de bloques paralelos analizando las relaciones entre altura, orientación, asoleamiento y densidad. Más tarde, Lionel March a partir de esos principios demostró que, manteniendo la garantía del asoleamiento en una distribución de bloques paralelos, hay un límite máximo para la densidad, cualquiera que sea la altura, que es de 1.333 hab/ha (March, 1975). Al otro lado del Atlántico, Wright proyecta su Broadracre City, una ciudad extendida y dispersa por un territorio bajo el signo de la descentralización de actividades, la red de autopistas, y la baja densidad residencial: a cada familia un acre de tierra (2,5 viv/ha netas).

En cierto modo, Boadracre era una profecía de lo que iba a ocurrir en las grandes ciudades americanas de la segunda mitad del siglo cuando emerge con fuerza el fenómeno del sprawl residencial unido a la descentralización de actividades y empleos, fenómeno que pronto atraería la atención de diferentes especialistas ante la magnitud de los costes directos e indirectos que esa forma de crecimiento estaba produciendo.

En la valoración del papel de la densidad en la forma y condiciones funcionales y de explotación de los desarrollos residenciales, confluyen diferentes enfoques que desde mediados del siglo XX han contribuido a la consolidación de la noción de ciudad compacta. Estas diferentes visiones se pueden agrupar en cuatro líneas: densidad e imagen interpretativa del territorio, densidad y urbanidad, densidad y eficiencia económica, y densidad y eficiencia energética. En la actualidad, esta última línea es a la que se le está prestando más atención, dando lugar a un debate intenso por sus implicaciones con el transporte, sector clave en las estrategias de sostenibilidad.

Este artículo expone las ideas básicas que inspiraron estas diferentes líneas, deteniéndose con más detalle en el caso de la implicación de la densidad con la movilidad a través de los resultados de un trabajo de investigación realizado sobre el territorio metropolitano de Alicante-Elche.

\section{Densidad e imagen interpretativa}

Como se ha comentado, desde finales de los años sesenta del siglo pasado se comienza a tomar conciencia en Europa de la emergencia de un crecimiento urbano de 
baja densidad que atrae la atención de estudiosos de la ciudad y el territorio, los que plasmarán sus reflexiones en imágenes interpretativas del fenómeno, sin descender a un plano más analítico y cuantitativo.

Henri Lefebvre (1972) ya escribe sobre un "tejido urbano" que se extiende por el medio rural, generando nuevas formas espaciales y sociales que pone en crisis el binomio antagónico tradicional ciudad-campo. Desde entonces se irán sucediendo diferentes aportaciones dirigidas a encontrar imágenes interpretativas de los procesos de crecimiento urbano difuso de baja densidad. En 1968 G. Samoná propone la noción de campo urbanizado para nombrar los nuevos fenómenos territoriales, sosteniendo incluso que esos desarrollos bien ordenados podrían constituir una alternativa a la congestión de las ciudades. Por la misma época aparece en la literatura geográfica francesa la noción de periurbanización, para designar los procesos de urbanización discontinua más allá de los límites de la ciudad y donde predomina el hábitat individual ${ }^{4}$. Una imagen interpretativa que ha tenido mucho éxito es la ciudad difusa enunciada por F. Indovina (1990), aunque poco antes, G. Piccinato había propuesto la de urbanización difusa.

\section{Densidad y urbanidad ${ }^{5}$}

En nuestra opinión, la primera descripción -aunque no la denomine así- de las características de la ciudad compacta como tejido denso y complejo aparece en los contenidos de la obra “Muerte y Vida de las Grandes Ciudades”, publicada en 1961, de la que es autora Jane Jacobs. En ella, su autora, desde la observación y el sentido común, discute el urbanismo de los expertos y urbanistas seguidores del Movimiento Moderno que habían sido incapaces de conseguir en sus realizaciones residenciales la recreación de la animación y vitalidad que encontramos en otras ciudades no planificadas.

En su libro, Jane Jacobs establece que entre las cuatro condiciones que deben darse en un sector residencial para garantizar una vida urbana intensa, una de ellas debe ser la necesidad de una cierta densidad:

"El distrito ha de tener una concentración de personas suficientemente densa, sea cual fuere el motivo que les impulsa a las diferentes personas a ocuparlo. Natu-

En 1975 el informe Mayoux reconoce el fenómeno de la periurbanización (Barattucci, 2003).

5 Entendemos la noción de urbanidad, siguiendo a F. Choay (1994), como un indicador cualitativo del grado de relación entre la forma urbana física y la forma social. La Bienal de París de 1980 reunió a un grupo de arquitectos y urbanistas en torno al tema de la urbanité/urbanity, y en el preámbulo de la convocatoria se definía así a la urbanidad: En la Edad Media, urbanidad significaba el gobierno de la ciudad; más tarde se entiende por esta palabra todo lo que tenga carácter urbano. En los tiempos modernos tiene nuevos significados: agradable, servicial, cortés, que implica un saber vivir y una experiencia del mundo. Un nuevo uso de este término ha aparecido recientemente e indica los varios aspectos de la organización y creación urbana que se ha desarrollado como reacción a los estragos provocados por las prácticas del urbanismo moderno (desde la posguerra hasta la actualidad) y a las desviaciones tecnocráticas nacidas de las doctrinas funcionalistas que favorecen los aspectos mecanizados, cualitativos y materiales de la ciudad. De hecho, los métodos del llamado urbanismo moderno han dirigido la segregación de las ciudades y la fragmentación del tiempo y del espacio. Este ideario ha hecho emerger un fuerte sentido de alienación y ha contribuido a la pérdida de la identidad de la ciudad. En una tentativa para liberar al urbanismo de estas desagradables asociaciones se ha sugerido un nuevo uso de la urbanidad que enfatizaría el uso y experiencia de la ciudad así como su potencial arquitectónico. Urbanidad, como un urban savoir faire y urban savoir vivre (Revista Architectural Design, Vol. 1, No. 12 1980). 
ralmente incluimos en este concepto los residentes de manera regular en el mismo" (Jacobs, 1973).

“¿Cuál es la densidad de viviendas apropiada para una ciudad?”. Se pregunta en otro lugar de su obra, estableciendo las 100 viv/acre neto (250 viv/ha neta) como cifra óptima a la que llega por observación y experiencia de situaciones en diferentes ciudades americanas contemporáneas:

"En los lugares en los que el promedio caiga claramente por debajo del centenar de viviendas por acre, la vitalidad queda muy menguada" (Jacobs, 1973).

Lógicamente, también se hacía la pregunta pertinente “¿Cuándo una densidad alta es demasiado alta?”. Aunque establece ese límite en 200 viv/acre neto ( 500 viv/ha neta), nos advierte de que el aumento de densidad residencial implica tipologías de edificación cada vez más homogéneas que impiden la diversidad visual del espacio urbano.

Para Jane Jacobs, la densidad es una variable necesaria pero no suficiente para generar la vida urbana, se necesitan otras condiciones complementarias que van desde la existencia de usos no residenciales a la limitación de espacio libre (sostiene que el máximo de este último no debe superar el 70 \%) con bloques de corta longitud de fachada y espacios públicos en localizaciones adecuadas para garantizar su uso ${ }^{6}$.

El ideario urbanístico de Jane Jacobs, a pesar de su posición favorable a las grandes ciudades, se reduce a la escala del barrio. Lo mismo se puede decir del contenido del "Manifiesto para el diseño urbano", en el que Donald Appleyard y Alan Jacobs sostienen, desde posiciones fundadas en la reintroducción de la unidad vecinal, que "la densidad de población justificará la existencia o no de ciertos usos y servicios que consideramos importantes para la vida de la ciudad", cuantificando unos límites de densidades máximas y mínimas para garantizar la generación de vida urbana de 120 y 40 viv/ha respectivamente (Appleyard y Jacobs, 1984).

El mismo año en que se publica el libro de Jane Jacobs aparece otro tan importante como el suyo por sus aportaciones al urbanismo del siglo XX, nos referimos a "La Ciudad en la Historia" del que es autor Lewis Mumford, un autor imprescindible para entender la urbanística norteamericana de la primera mitad del siglo XX. Mumford -muy vinculado al movimiento del Regional Planning Association of América-, desde una visión más territorial que la de Jane Jacobs, alertaba sobre los efectos del sprawl en las ciudades americanas:

"Incluso en el caso de una ciudad tan espaciosa como Washington, sólo la inicial zona central tiene una densidad residencial de veinte o más familias por hectárea: en los difusos suburbios el promedio está por debajo de veinte y una veloz oleada está poniendo una zona aún mayor bajo una densidad de menos de diez familias por hectárea. Esto es ruinoso para el modo de vivir urbano y para la recreación en las horas de ocio; pues la tentativa de establecer autopistas que sirvan a las zonas distantes no sólo esterilizará cada vez más la tierra, sino que determinará servicios sociales que deberían estar concentrados en nuevas ciudades, organizados de tal modo que difundan y amplifiquen los servicios centrales" (Mumford, 1966).

6 Aplicando ese $70 \%$ de suelo libre a un sector residencial con una densidad óptima neta de 250 viv/ha (óptima según Jane Jacobs), obtenemos una densidad bruta del orden de 75 viv/ha, que es el máximo que establece la legislación española del suelo para nuevos desarrollos residenciales. 
Si la noción de "urbanidad" está ligada a la de "forma de la ciudad", no podemos cerrar este apartado sin hablar de las aportaciones de Kevin Lynch a la cuestión. Lynch sostiene que no se puede confiar solamente en los indicadores cuantitativos para definir lo que él llama "la buena forma" de la ciudad, sino que además ésta se constituye a través de la experiencia de los lugares, una experiencia que va más allá de la percepción que nos inducen los sentidos. En 1960 publicó "La Imagen de la Ciudad" en donde establecía un vocabulario para "leer" el paisaje urbano fundado en la psicología de la Gestalt (Lynch, 1960). Sin embargo, varios años después fue enriqueciendo ese planteamiento inicial dándole mucho más protagonismo a cuestiones relacionadas con el significado, como la noción de "sentido" de los lugares, más ligada a la memoria que a lo sensorial. Para Kevin Lynch nos encontramos en un buen lugar cuando la expresión "estoy aquí" equivale a "yo soy". A pesar de que Lynch no se posicionó de un modo claro en contra del sprawl, esa buena experiencia de los lugares difícilmente se puede sentir en desarrollos urbanos de baja densidad y de forma difusa (Lynch, 1981).

Sin embargo, también encontramos posiciones que sostienen, por el contrario, que en el suburbio está surgiendo una nueva urbanidad ligada al progreso tecnológico y a las comunicaciones. Para Melvin Weber, la concentración de la población en un territorio era algo circunstancial derivado de la fricción del espacio para la interacción social, pero cuando el espacio es vencido por la velocidad y las telecomunicaciones no hay razón que justifique la aglomeración ${ }^{7}$. Como ha apuntado M. Solá-Morales (1997), las tesis de Weber traían una visión optimista de la dispersión territorial y alentaban a una superación del modelo urbano compacto.

\section{Densidad y eficiencia económica}

El fenómeno del sprawl, la suburbanización de baja densidad en las ciudades americanas, se inició tras la Segunda Guerra Mundial por la confluencia de diversos factores, entre los que cabría destacar las facilidades para la compra de viviendas y coches unido a las ventajas ofrecidas a la movilidad privada por las nuevas redes de autopistas, así como el bajo precio del carburante ${ }^{8}$. El sprawl americano ha sido definido del modo siguiente: "Una forma de urbanización en fragmentos continuos o discontinuos, ejes comerciales a lo largo de carreteras, baja densidad, actividades y usos de suelo separados y aislados, modo de transporte predominante en coche y mínimo espacio público abierto" (Gillhan, 2002).

7 En Weber (1970) ya se encuentra una visión desterritorializada del fenómeno urbano: "un dominio urbano no es ni un asentamiento ni un territorio sino el ámbito de comunicación de grupos humanos heterogéneos a través del espacio".

8 En las ciudades de los Estados Unidos, durante los años setenta el 95\% del crecimiento de la población y el 66\% del de los empleos se concentraba en los suburbios. En 1990 el 62\% de todos los empleos de la nación estaban en los suburbios (Gillhan, 2002). 
CUADRO 1 | Evolución de la población del centro y de los suburbios en ciudades norteamericanas, 1950-2000 (en millones)

\begin{tabular}{|c|c|c|c|c|c|c|c|c|c|c|c|c|}
\hline & \multicolumn{2}{|l|}{1950} & \multicolumn{2}{|l|}{1960} & \multicolumn{2}{|l|}{1970} & \multicolumn{2}{|l|}{1980} & \multicolumn{2}{|l|}{1990} & \multicolumn{2}{|l|}{2000} \\
\hline & POBLACIÓN & $\%$ & POBLACIÓN & $\%$ & POBLACIÓN & $\%$ & POBLACIÓN & $\%$ & POBLACIÓN & $\%$ & POBLACIÓN & $\%$ \\
\hline CENTRO & 49,7 & 59 & 58 & 51 & 63,8 & 46 & 72,4 & 42 & 77,8 & 40 & 85,4 & 38 \\
\hline SUBURBIOS & 35,2 & 41 & 54,9 & 49 & 75,6 & 54 & 99,3 & 58 & 114,9 & 60 & 140,6 & 62 \\
\hline TOTAL & 84,9 & 100 & 112,9 & 100 & 139,4 & 100 & 171,7 & 100 & 192,7 & 100 & 226 & 100 \\
\hline
\end{tabular}

FUente O. Gillham (2002).

En los años cincuenta del siglo pasado aparecen diferentes trabajos que se dirigen a determinar los costes de la dispersión residencial emergente en términos de inversión de capital, así como en costes de explotación de los servicios públicos que demandan los territorios suburbanos. Estos estudios suelen coincidir en que los desarrollos difusos son más "caros" que los compactos (Wheaton y Schussheim, 1955). Se pueden encontrar trabajos en donde se demuestra cómo la alta densidad residencial reduce los costes de inversión y explotación relacionados con los servicios de policía, residuos, saneamiento y abastecimiento de agua, pero no de los de educación (Downing, 1977) ${ }^{9}$. En estos primeros estudios que aparecen antes de la crisis del petróleo de mediados de los setenta no aparecen todavía de un modo relevante los costes de transporte asociados a las variaciones de densidad.

La densidad como un indicador de la intensidad de edificación que se puede alcanzar en una parte del territorio, está también muy vinculada a la repercusión de los costes de suelo y construcción en los de la vivienda. En Gran Bretaña, en un informe gubernamental en 1962, se recomendaban densidades residenciales entre 12 y 20 viv/acre ( 30 y 50 viv/ha aproximadamente), sosteniendo que por encima de las 50 viv/ha los costes de construcción no compensaban el ahorro en los costes de suelo (Lever, 1962). Estas recomendaciones aparecen en un momento en que se están proyectando y construyendo un gran número de sectores residenciales, muchos de ellos de vivienda social. Hay que citar en este punto las aportaciones de L. Martin y L. March (1975) que realizaron en esa época interesantes trabajos acerca de las relaciones entre tipologías y aprovechamiento de suelo, así como sobre la forma óptima de la edificación con relación a su coste de construcción y ahorro energético.

\section{Densidad y eficiencia energética}

Podemos situar el arranque de la difusión de la conciencia medioambiental en la publicación en 1972 del controvertido Informe Meadows. Su publicación coinci-

9 Herce y Magriñá (2002) sostienen que, en general, los costes de urbanización no varían entre densidades altas y bajas. Cuando la densidad se triplica, el incremento del coste puede estar en torno al 20\%, y el servicio más sensible es el abastecimiento de energía eléctrica. 
dió con la primera gran crisis del petróleo que, aunque motivado por las tensiones árabe-israelitas, abrió un futuro de crisis energética derivado tanto de la fragilidad del abastecimiento como de la escasez de los recursos energéticos basados en combustibles fósiles.

En realidad, el Informe Meadows no alertaba tanto sobre la degradación del medio ambiente como sobre el agotamiento de los recursos naturales en el caso de que se mantuviese un crecimiento tendencial del modelo económico dominante en Occidente. En cualquier caso, el debate acerca de la incompatibilidad entre desarrollo económico y medio ambiente estaba abierto. Algunos años después, en 1987, la Comisión Bruntland definió la noción de desarrollo sostenible basado en un diálogo entre desarrollo económico y medio ambiente como una exigencia ética, y que en cierto modo se planteaba como una alternativa al alarmismo del informe del Club de Roma. En la nueva noción de sostenibilidad el transporte aparece como uno de los sectores claves en el que confluyen desarrollo económico y modo de vida con consumo energético, con mayor relevancia desde que en los años noventa se comenzase a extender la evidencia de la relación entre emisiones de $\mathrm{CO}^{2}$ y calentamiento global.

A finales de los ochenta, las investigaciones de Newman y Kenworthy sobre las relaciones entre consumo de combustible y formas de desarrollo espacial les llevó a la definición de ciudad compacta como forma más eficiente para el consumo energético en el transporte, al encontrar evidencias empíricas de que este último aumentaba al bajar la densidad y viceversa. Estos autores argumentaban que las ciudades europeas son más densas que las norteamericanas y australianas, lo que asociaban a un uso elevado del transporte público y, en consecuencia, un bajo consumo de carburante per cápita. La media de consumo de combustible para coches en esas ciudades americanas era casi cuatro veces más alto que en las ciudades europeas y el doble que en las australianas. También concluían que ciudades con concentración de empleos y un buen sistema de transporte público acorde con esas localizaciones contribuía a una tasa más baja de consumo de energía frente a los casos de ubicación de empleos dispersos (Newman y Kenwoththy, 1989).

Las tesis de Newman y Kenworthy han sido discutidas metodológica e ideológicamente, entre otros, por Gordon y Richardson, quienes en un conocido artículo afirmaban que aquellos autores se fundaban en análisis defectuosos y que las políticas de planeamiento propuestas no eran viables. Para estos críticos no se puede generalizar la relación entre densidad y movilidad, sosteniendo que en las ciudades americanas la localización periférica de los empleos y próxima a los suburbios reducía la longitud de los viajes; por otro lado, también argumentaban que los hábitos de comportamiento con relación a la movilidad de los ciudadanos en áreas densas pueden contradecir la evidencia de bajo uso del vehículo privado (Richardson y Gordon, 1997) ${ }^{10}$.

10 En Toronto hay una importante concentración de empleo en torno al Pearson Airport situado en plena zona Metro de la periferia suburbana. De casi 40.000 empleos, el 52\% corresponde a trabajadores que residen en esa zona periférica y, aunque los desplazamientos puedan ser más cortos para éstos, el porcentaje de viajes en transporte público y andando es sólo del 4\% (Toronto Urban Development Services, 1998). 
FIGURA 1 | Densidad de población y consumo de energía en diferentes ciudades del mundo

Consumo de energía per capita (1000 millones de joules)

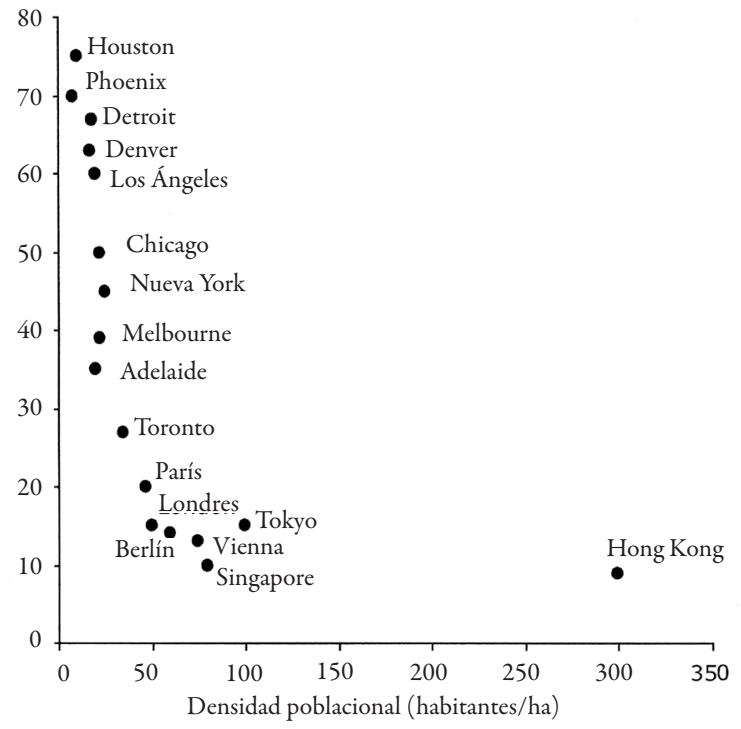

fuente Agencia Europea del Medio Ambiente (2006).

CUADRO 2 | Modo de transporte y densidad

\begin{tabular}{|c|c|c|c|c|}
\hline Continente & Ciudad & $\begin{array}{l}\text { VIAJES TRANSPORTE } \\
\text { PÚBLICO (\%) }\end{array}$ & $\begin{array}{l}\text { VIAJES A PIE/ } \\
\text { BICICLETA (\%) }\end{array}$ & $\begin{array}{l}\text { DENSIDAD } \\
\text { HABITANTES/ } \\
\text { HECTÁREA }\end{array}$ \\
\hline \multirow[t]{4}{*}{ Norteamérica } & Houston & 4 & 3 & 11,71 \\
\hline & Los Ángeles & 7 & 4 & 29,00 \\
\hline & Chicago & 15 & 5 & 48,04 \\
\hline & Nueva York & 27 & 7 & 92,57 \\
\hline \multirow[t]{3}{*}{ EUROPA } & Frankfurt & 42 & 9 & 61,6 \\
\hline & Estocolmo & 55 & 14 & 92,61 \\
\hline & París & 36 & 15 & 97,66 \\
\hline \multirow[t]{3}{*}{ Asıa } & Tokio & 49 & 22 & 133,32 \\
\hline & Seúl & 60 & 20 & 301,78 \\
\hline & Manila & 54 & 18 & 376,12 \\
\hline
\end{tabular}

FUENTE NEWMAN y KENWORTHY (1999) y ELABORACIÓN PROPIA. 
En cualquier caso, en la actualidad los modelos de planeamiento urbano y territorial se dirigen a desarrollar propuestas fundadas en la densidad media y alta así como en el "mix" funcional dirigido al ahorro energético y a la reducción de emisiones en tres líneas estratégicas: limitación y reducción del uso del coche privado, limitación de la distancia recorrida y limitación del número de viajes.

\section{Densidad y movilidad en el área urbana de Alicante ${ }^{11}$}

El territorio metropolitano de Alicante-Elche ha sido históricamente un espacio muy acotado aunque poco vertebrado, social y económicamente, lo que unido a sus diferencias funcionales y espaciales -turismo, servicios al norte e industria al sur como sectores económicos hegemónicos- ha generado un territorio bipolar alrededor de dos núcleos dominantes en torno a los cuales se localizan los municipios de San Vicente, San Juan, Campello y Muchamiel, al norte; y Crevillente y Santa Pola, al sur.

Como hemos demostrado mediante el análisis de los flujos de tráfico con motivo trabajo, las dos áreas presentan un alto grado de autonomía, es decir, que los flujos entre las áreas urbanas de Alicante y Elche son poco relevantes en comparación con los viajes internos en cada una de ellas (Casado, no publicado) ${ }^{12}$. Esta autonomía relativa de los dos espacios que componen el territorio metropolitano de AlicanteElche legitima el análisis y diagnóstico que hemos hecho para la zona norte.

El área urbana de Alicante la definimos como un tejido continuo extendido por los términos municipales contiguos, y en donde la presencia dominante del núcleo central es compartida por un incipiente policentrismo que se detecta por el creciente proceso de localización de empleos y servicios en los municipios del área, como en Campello y, especialmente, San Vicente ${ }^{13}$. En la formación del tejido territorial del área ha predominado la forma dispersa de baja densidad residencial. En los años 60 y 70 del siglo pasado se inició un proceso de crecimiento extensivo de dispersión y agregación, facilitado, en primer lugar, por las condiciones de accesibilidad que proporcionaban la red de carreteras y caminos y, posteriormente, por efecto metastático inducido por las piezas y tejidos residenciales de baja densidad existentes. En la actualidad, del orden de un 50\% de los residentes en el área viven en un territorio con una densidad en torno a las 10 viv/ha. Un indicador del grado de compacidad

11 La información básica de movilidad ha sido extraída del estudio "El transporte metropolitano del área de Alicante. Encuesta domiciliaria de movilidad" (Generalitat Valenciana, 2001). La explotación de las relaciones entre variables de movilidad y urbanísticas ha sido realizada por Maria José Ivorra Ivars en su proyecto de fin de carrera de la titulación de Ingeniero de Caminos, Canales y Puertos, dirigido por J. R. Navarro Vera y A. Ortuño Padilla.

12 También, según los datos aportados por el Censo de 2001, sólo 3.844 ocupados en el municipio de Alicante trabajan en Elche siendo el total de 115.256 ocupados, es decir, sólo un 3,3\%, mientras que en sentido inverso 2.446 de un total de 80.334, o sea, un 3\%. Como ejemplo de mayor integración, el 6\% de los residentes en Gijón -5.996 sobre 99.695trabajan en Oviedo.

13 Así, el Índice de Actividad Económica per cápita relativizado por el número de habitantes ha crecido más en términos relativos en Campello y San Vicente que en Alicante entre 2002 y 2007. El Índice de Actividad Económica, referido a 2007 y 2002, se obtiene en función del impuesto correspondiente al total de actividades económicas empresariales (industriales, comerciales y de servicios) y profesionales. Es decir, incluye todas las actividades económicas excepto las agrarias (que no están sujetas al impuesto de actividades económicas). El valor del índice expresa la participación de la actividad económica (en tanto por 100.000) de cada municipio, provincia o comunidad autónoma sobre una base nacional de 100.000 unidades equivalente a la recaudación del impuesto de actividades económicas empresariales y económicas (La Caixa, 2009). 
del crecimiento urbano es la relación entre las magnitudes de crecimiento de suelo urbanizado y población, que en el territorio del término municipal de Alicante para el periodo 1995-2000 es del 44\% para el suelo y del 9\% para la población.

En la forma del territorio que constituye el ámbito de esta investigación el planeamiento urbano de los municipios que lo constituyen ha tenido un papel muy relativo. Únicamente el control público del crecimiento de los tejidos territoriales comenzó a surtir efectos hacia los años 80 del siglo pasado, cuando ese proceso había comenzado más de veinte años antes. A continuación, exponemos una síntesis del desarrollo de ese proceso en el territorio marco de nuestra investigación, iniciado en 1956, fecha de la aprobación de la primera legislación española estatal de ordenación urbana y de gestión del suelo.

Fase I. $1956-1978$

En esta fase, en la que comienzan a surgir ocupaciones de suelo inducidas por el turismo, podemos encontrar dos modelos de crecimiento: uno, fragmentado y disperso de vivienda unifamiliar de baja densidad hacia el interior del territorio, ligado a la accesibilidad que aporta la red de caminos y carreteras locales y provinciales; y otro, más denso y compacto, vinculado a la costa en el entorno de municipios, como los que surgen a lo largo del borde litoral norte de la ciudad de Alicante.

Esta fase termina con la entrada en servicio de la autopista A-7 que discurre bordeando la costa mediterránea de la península. Esta infraestructura rompe el modelo de accesibilidad territorial radiocéntrico introduciendo un potente eje lineal que influirá en los desarrollo de los asentamientos futuros.

En esta fase, el crecimiento de los tejidos territoriales fue "espontáneo". En los municipios no existían planes de ordenación, y su ausencia se sustituía por unas normas de ámbito provincial muy escuetas y dirigidas a un cierto control de la edificación más que al del urbanismo.

\section{Fase II. 1979 - 1990}

El año escogido para el inicio de esta fase coincide con el comienzo de los primeros gobiernos municipales democráticos tras la guerra civil, y que tuvieron, entre sus tareas prioritarias, comenzar a reequipar los déficits en dotaciones y servicios públicos y poner orden y disciplina en el planeamiento de las ciudades españolas tras cuarenta años de dictadura. Para la ordenación urbana y del territorio, los municipios contarán con un nuevo instrumento legal, una reforma de la ley del 56 que se había aprobado en 1975. Sin embargo, la nueva situación política y legal no tendría efectos apreciables en el territorio hasta la década siguiente.

\section{Fase III. 1990 - 1994}

La última década del siglo comenzó con una coyuntura de crisis económica que frenó el crecimiento de la urbanización del territorio. En estos años se comenzó a extender con cierto rigor el control del desarrollo del suelo en los municipios de área de nuestra investigación que poco a poco se habían ido dotando de planes munici- 
pales de ordenación urbana. El Plan General de Ordenación de la ciudad de Alicante aprobado a finales de los ochenta era muy restrictivo en la oferta de suelo urbanizable y estaba dirigido básicamente hacia lo que se denominó en la época "terminar y completar la ciudad". Planes como éste sí tuvieron una influencia positiva en la racionalización del crecimiento territorial.

Esta fase cierra el proceso histórico de formación y consolidación del territorio que constituye el ámbito espacial de nuestro estudio. No obstante, queremos añadir algo más. El año 1994 es muy significativo en el urbanismo de esta región autónoma, porque fue aprobada una nueva ley de ámbito regional que pretendiendo agilizar el planeamiento y la gestión del suelo desembocó en una verdadera desregulación del planeamiento que, coincidiendo con la recuperación económica de principio de este siglo, ha tenido efectos muy negativos en el territorio ${ }^{14}$.

FIGURA 2 | Territorio norte del área metropolitana Alicante-Elche. Distribución de densidades

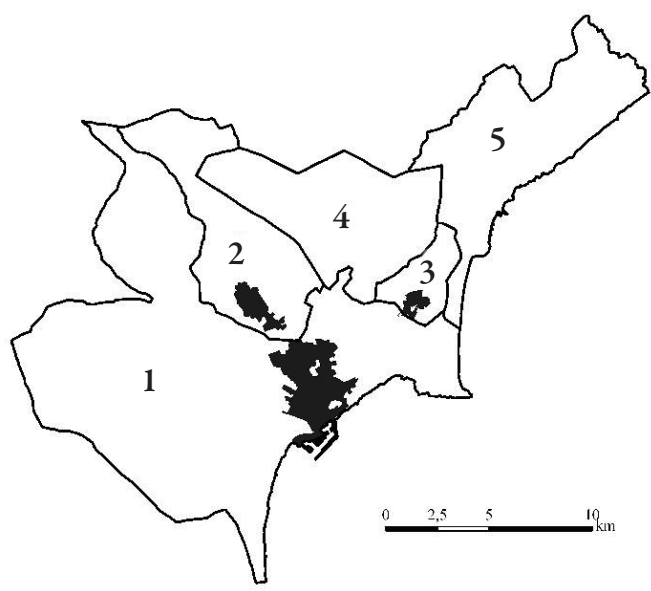

1. Alicante

2. San Vicente del Raspeig

3. San Juan

4. Muchamiel

5. Campello
$\mathrm{D}($ media $)=50,37$ viv $/$ ha $(\sigma=16)$

$\square \mathrm{D}($ media $)=9,5 \mathrm{viv} / \mathrm{ha}$ $(\sigma=8,1)$

FUENTE ELABORACIÓN PROPIA.

Los resultados más relevantes de nuestra investigación confirman que algunos de los indicadores de movilidad están fuertemente correlacionados con la densidad residencial a lo largo de este territorio, como resumimos a continuación:

14 Se trata de la Ley 6/1994 del Gobierno de la Generalitat Valenciana, denominada Ley Reguladora de la Actividad Urbanística. 
1. Las zonas más densas del territorio generan más viajes en todos los modos que las menos densas. Por tanto, el índice de movilidad total (viajes/hab/día) aumenta a medida que lo hace la densidad. En cambio, para densidades bajas aumenta la generación de viajes en vehículo privado con relación a los generados en las zonas densas.

FIGURA 3 | Correlación entre el número de viajes/habitante/día y la densidad de viviendas

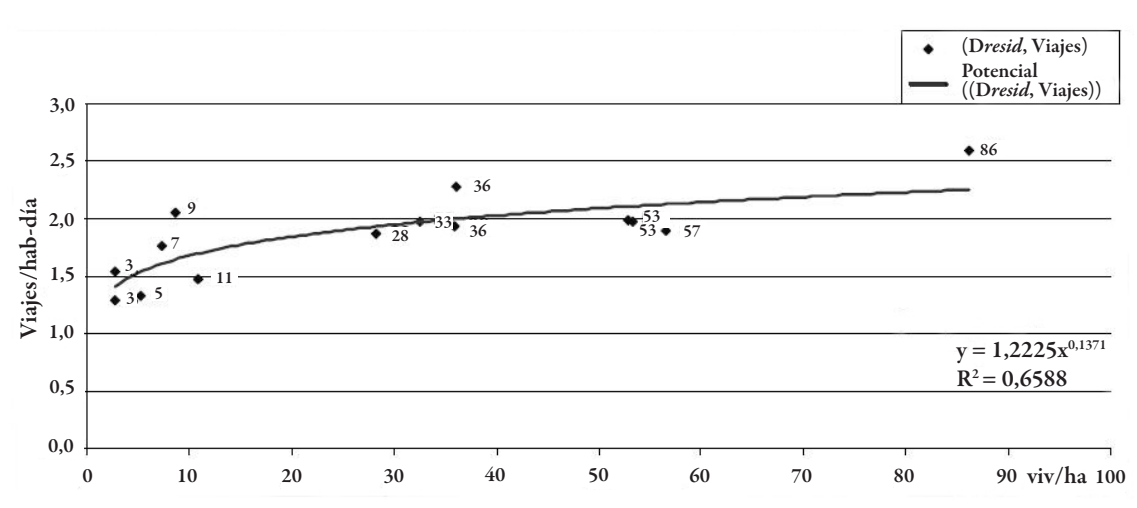

FUENTE ELABORACIÓN PROPIA.

FIGURA 4 | Correlación entre los viajes generados por el transporte privado y la densidad residencial

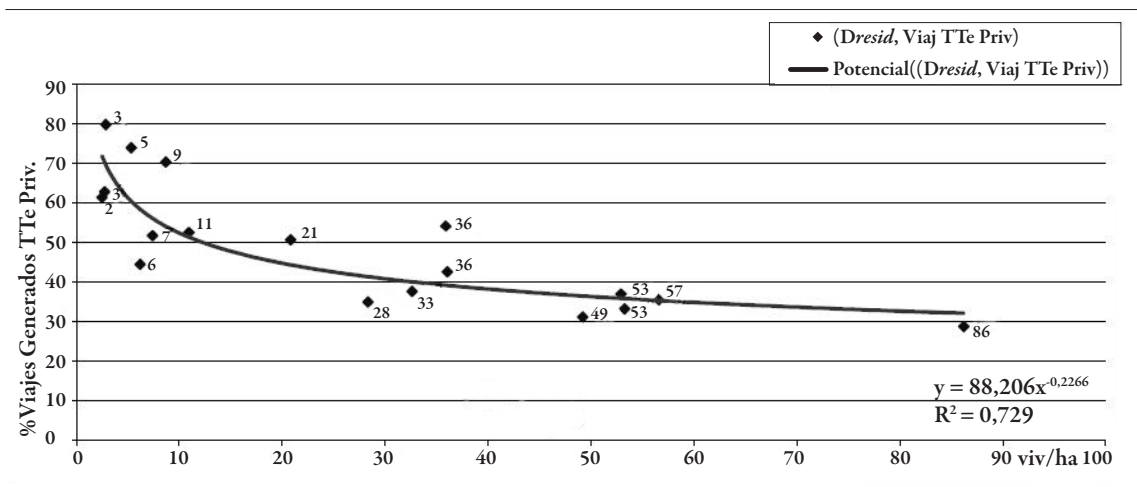

FUENTE ELABORACIÓN PROPIA.

2. Hay una relación inversa entre el nivel de motorización (vehículos/1.000 hab) y la densidad. La diferencia del nivel de motorización es muy alta, ya que encontramos zonas con valores de éste próximos a 500 en las de baja densidad y a menos de 300 en las de alta densidad ${ }^{15}$.

15 En una investigación realizada en ciudades japonesas se encontró una correlación análoga a la obtenida en la nuestra en la relación entre densidad e índice de motorización (Kaido, 2006). 
FIGURA 5 | Correlación entre el nivel de motorización y la densidad residencial

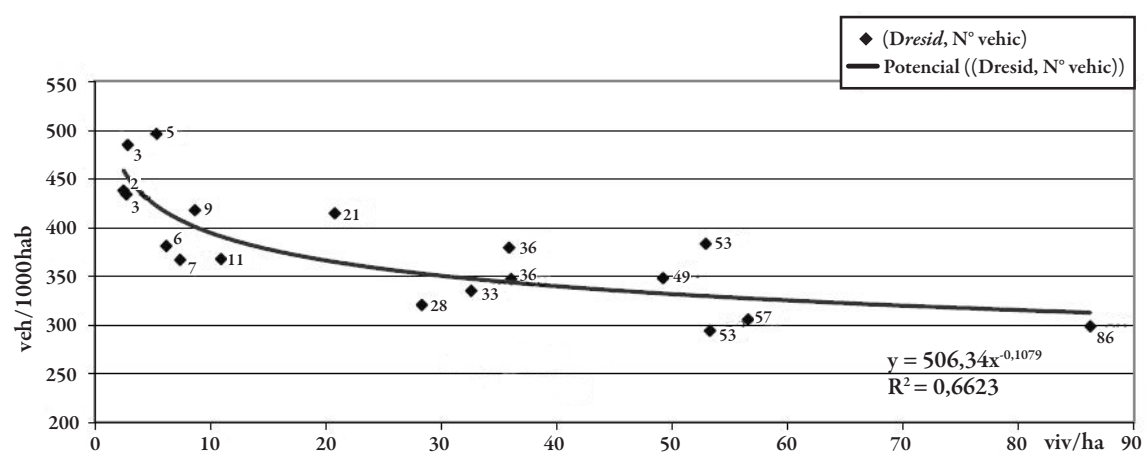

FUENTE ELABORACIÓN PROPIA.

3. El estudio confirma la tendencia observada en otros lugares a la disminución del número de desplazamientos a pie con la disminución de la densidad y viceversa ${ }^{16}$.

FIGURA 6 | Correlación entre los viajes generados a pie y la densidad residencial

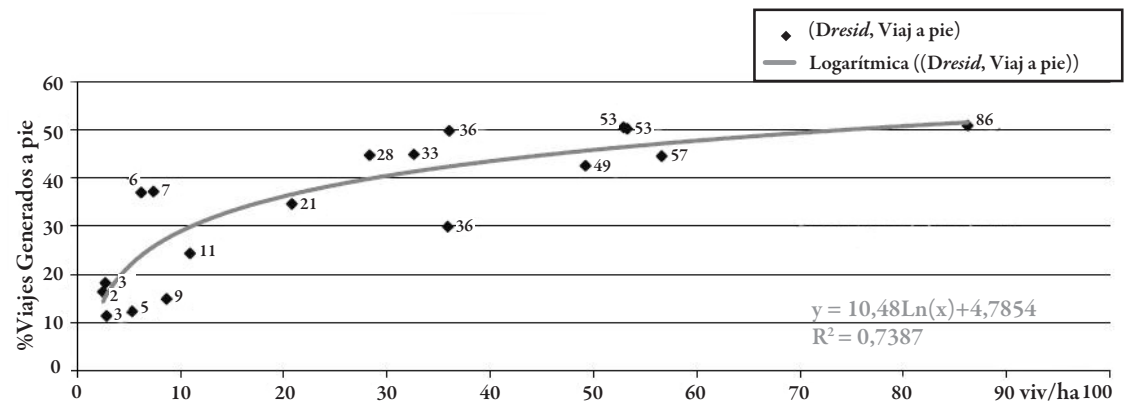

FUENTE ELABORACIÓN PROPIA.

4. Finalmente, se ha verificado la evidencia empírica que relaciona de un modo directo la atracción de tráfico con número de empleos localizados en cada zona.

16 En una reciente investigación en ciudades y áreas metropolitanas americanas se cruzaron indicadores de densidad residencial, movilidad peatonal y obesidad. A pesar de que la relación no es absolutamente lineal, el estudio concluye que hay una relación inversa entre presencia de personas con obesidad con la densidad y la movilidad peatonal, mientras que estas dos últimas variables tienen una relación directa (Ewing, Schmid, Killingsworth, Zlot y Raudenbush, 2008). 
FIGURA 7 | Correlación entre los viajes generados por el motivo trabajo y el número de empleados

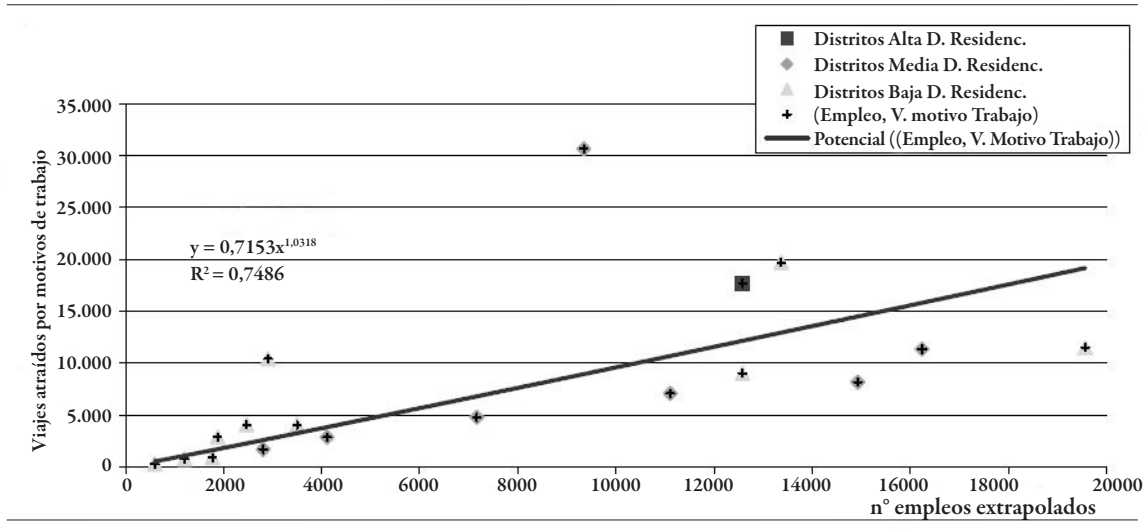

FUENTE ELABORACIÓN PROPIA.

Las curvas de correlación obtenidas suelen ser exponenciales, presentan gradientes bajos y homogéneos para los valores más densos mientras que a partir de un entorno de las $30 \mathrm{viv} /$ ha hacia las bajas densidades se producen los cambios de gradientes más altos, lo que interpretamos como que es en las zonas de baja densidad donde se produce la relación más intensa entre ésta y los indicadores de movilidad.

CUADRO 3 | Resumen de relaciones entre densidad residencial y variables de movilidad en el área urbana de Alicante $\sigma=$ desviación estándar

\begin{tabular}{l|c|c|c|c|c}
\hline & $\begin{array}{c}\text { DENSIDAD } \\
\text { MEDIA }\end{array}$ & $\begin{array}{c}\text { NÚMERO VIAJES } \\
\text { /HABITANTE } \\
\text { Y DÍA }\end{array}$ & $\begin{array}{c}\text { \% VIAJES A } \\
\text { PIE }\end{array}$ & $\begin{array}{c}\text { \% VIAJES EN } \\
\text { TRANSPORTE } \\
\text { PRIVADO } \\
\text { GENERADOS }\end{array}$ & $\begin{array}{c}\text { NIVEL } \\
\text { MOTORIZACIÓN. } \\
\text { VEHÍCULOS } \\
/ \text { I.00o } \\
\text { HABITANTES }\end{array}$ \\
\hline $\begin{array}{l}\text { Áreas con Densidad } \\
>30 \text { viviendas/hectárea }\end{array}$ & $50,37(\sigma=16)$ & $2,31(\sigma=0,6)$ & $45,25(\sigma=6,5)$ & $37,62(\sigma=7,41)$ & $336(\sigma=34,54)$ \\
$\begin{array}{l}\text { Áreas con Densidad } \\
<30 \text { viviendas/hectárea }\end{array}$ & $9,5(\sigma=8,1)$ & $1,87(\sigma=0,84)$ & $\begin{array}{c}25,0(\sigma= \\
11,76)\end{array}$ & $58,2(\sigma=13,99)$ & $404(\sigma=48,21)$ \\
\hline
\end{tabular}

FUENTE ELABORACIÓN PROPIA.

No se puede olvidar que estos resultados se inscriben en unas condiciones espaciales y funcionales específicas para el territorio estudiado que puede afectar los resultados, como la oferta de transporte público. En nuestro estudio, la densidad, a diferencia de otros, no explica de modo significativo las variaciones en la generación de viajes en transporte público, lo que se debe a una oferta irregular y de calidad media o baja en ese territorio. 


\section{Conclusiones}

La noción de ciudad compacta es un concepto basado, en ocasiones en las que se defiende este modelo, en suposiciones o en un imaginario urbano no exento de cierta nostalgia de la ciudad mediterránea tradicional. Es cierto que hay evidencias empíricas -y nuestra investigación las afirma- que muestran que el modelo compacto con mix funcional puede garantizar mejores condiciones de sostenibilidad ambiental. Sin embargo, la relación entre forma urbana y eficiencia energética está afectada por factores políticos, sociales y económicos que impiden una generalización del modelo compacto basado en la densidad media-alta.

Podemos encontrar tejidos urbanos compactos en áreas centrales de ciudades que presentan un perfil demográfico de envejecimiento junto con un perfil económico de renta media o baja, factores ambos determinantes de las condiciones de movilidad, en especial con relación al reparto modal y al índice de posesión de coches. Por otro lado, en los nuevos sectores residenciales de periferia en ciudades españolas, de densidades medias o altas, ocupados por familias jóvenes con niños en las que son activos ambos cónyuges - una composición social que no favorece el uso del transporte público- no hay más que pensar en los viajes generados para llevar a los niños al colegio. Específicamente, en nuevas áreas residenciales de baja densidad vinculadas a campos de golf en el Levante español se ha obtenido un índice de movilidad -número de desplazamientos por persona y día laboral-, entre 1,15 y 1,30. Los índices anteriores se corresponden con bastante exactitud al de los viajes mecanizados/persona y día en diferentes capitales medias españolas como Córdoba, Granada, Pamplona o Alicante, y que oscilan entre 1,10 y 1,34 (Navarro y Ortuño, no publicado). En estas mismas ciudades, el índice de movilidad en todos los modos se encuentra entre 2,2 y 2,5 desplazamientos/hab/día. El menor número de desplazamientos totales parece relacionarse con una mayor coordinación de las actividades a realizar en un único desplazamiento, ya que prácticamente cualquier desplazamiento supone utilizar el vehículo privado a diferencia de lo que ocurre en el modelo de ciudad compacta.

En todo caso, no se puede olvidar que la descentralización de actividades económicas, especialmente comerciales de uso frecuente, dificulta el acceso a las personas de edades avanzadas por cuanto aumenta la dependencia del automóvil y dificulta su acceso como cautivos del transporte público y a pie.

Conviene situar la dialéctica compacto-difuso en relación a la escala del ámbito para el que se está estableciendo un diagnóstico o proyectando un nuevo crecimiento. En la escala local es posible definir las variables e indicadores determinantes para su evaluación ambiental (López, 2007). Sin embargo, son necesarios más estudios empíricos que determinen cuáles son los parámetros que hacen más eficiente una forma de crecimiento en la escala metropolitana. En este último sentido, determinar el papel de la localización de los empleos con relación a las viviendas es un factor esencial en la sostenibilidad energética de un tejido territorial, aunque existen discrepancias sobre esta cuestión. Según Gordon y Richardson, las tendencias de localizar empleos entre los suburbios residenciales de baja densidad como ocurre en ciuda- 
des americanas y canadienses disminuye la longitud de los viajes al trabajo, mientras que para Peter Hall, aún aceptando esa hipótesis, al mismo tiempo se produce una transferencia del transporte público al coche en los recorridos radiales (Hall, 1995). Peter Hall formó parte del grupo Urban Task Force, una iniciativa oficial del gobierno inglés dirigido a sentar las bases de un urbanismo sostenible. En dicho informe, dirigido por Richard Rogers, se recomienda para los nuevos desarrollos residenciales una densidad minima de 40 viv/ $/ \mathrm{ha}^{17}$.

En cualquier caso, no se pone en duda que las alternativas de crecimiento sostenible en una escala territorial deben estar articuladas por potentes redes de transporte público. El Transit Oriented Development (T.O.D.) vinculado a la estrategia del smart growth que tiene a la ciudad de Portland como su paradigma, organiza el territorio como un rosario de núcleos residenciales y mix funcional con densidades decrecientes desde la estación, y con distancias máximas a recorrer a pie en torno a una milla (Calthorpe, 1993) ${ }^{18}$.

También está surgiendo una recuperación de la idea de greenbelt como elemento estratégico con la finalidad, entre otras, de limitar el crecimiento. El star model propuesto para Berlin recoge principios tanto del greenbelt como del T.O.D. (Amati, 2008).

Para terminar esta breve revisión de alguna de las propuestas más relevantes en Norteamérica como alternativas al urban sprawl, hay que citar al Smart Growth y al New Urbanism, que aunque en ocasiones se suelen asociar, porque tienen algunas bases comunes, en esencia son bastante diferentes. El primero es una estrategia a escala de ciudad o incluso de un territorio más amplio que fija objetivos de sostenibilidad ambiental para su crecimiento y desarrollo. Portland (Oregón) se ha convertido en una de las ciudades paradigmáticas del Smart Growth. En cambio el New Urbanism está dirigido hacia la planificación de comunidades residenciales sostenibles con un cierto grado de aislamiento. Están concebidos como barrios idílicos para una población blanca y de renta homogénea con una arquitectura seudo-victoriana, georgiana o colonial ${ }^{19}$. La ciudad de Seaside -escenario de la película El show de Truman del director australiano Peter Weir- es una de las más conocidas.

Hay que insistir en la exigencia de aumentar nuestro conocimiento del funcionamiento de las nuevas formas de crecimiento urbano y territorial que haga posible establecer criterios válidos y contrastados para determinar su eficiencia ambiental en sentido amplio. Y hemos de insistir porque en los últimos años las políticas imperantes de desarrollo espacial no han estado fundadas en una base razonable de lo que podíamos llamar una ética del territorio. En la Comunidad Valenciana y en Murcia, entre comienzos de siglo y 2007, estaban en ejecución, se habían aprobado o estaban en tramitación, más de 170 operaciones residenciales con campos de golf, en localizaciones aisladas, que en conjunto tenían una capacidad del orden de 500.000

17 http://www.urbantaskforce.org/UTF_final_report.pdf

18 El T.O.D. recoge ideas, tradiciones y experiencias que van desde la Unidad Vecinal a los proyectos de los años sesenta para el crecimiento de Copenhague (Plan de los Dedos) o Washington.

19 Wakeman, R. (1999). États-Unis: New Urbanism. Revista Urbanisme, No. 308, sep/oct. 
viviendas con una densidad de entre 15 y 20 viviendas por hectárea de media (Navarro y Ortuño, no publicado).

Para finalizar con los análisis acerca de los efectos de las nuevas formas de crecimiento urbano y modelo territorial, se advierte muy necesario recuperar los estudios sobre densidad y eficiencia económica, ampliarlos y actualizarlos, así como otros que se podrían denominar de "territorio y fiscalidad", es decir, encaminados a que la tributación residencial sea acorde a los costes que la forma de desarrollo territorial genere; costes desde los ámbitos económico, cuyos resultados se deberán obtener de los referidos análisis sobre eficiencia económica, ambiental -en el sentido de consumo de recursos naturales no renovables y generación de efectos externos negativos- y social -en términos de cohesión o fragmentación social. En definitiva, esa tributación debería aplicarse bajo el principio de la internalización de costes económicos, ambientales y sociales. IEURE

\section{Referencias bibliográficas}

Agencia Europea del Medio Ambiente (2006). Urban sprawl in Europe. The ignored challenge. Copenaghe: EEA.

Amati, M. (Ed.) (2008). Urban Green Belts in the Twenty-first Century. Hampshire: Ashgate.

Apleyard, D. \& Jacobs, A. (1984). Hacia un manifiesto por el diseño urbano. Revista Ciudad y Territorio, Vol. 59, No. 60, pp. 69-78.

Barattucci, C. (2003). Du periurbain aux urbanisation dispersées. Revista Urbanisme, No. 329, pp. 77-81.

Bentham, J. \& Foucault, M. (1979). El Panóptico. En el ojo del poder. Barcelona: La Piqueta.

Calthorpe, P. (1993). The next American Metropolis. New York: Princeton Architectural Press.

Casado Díaz, J.M. (Ed.) (No publicado). Propuesta de clasificación territorial a efectos estadísticos de la Comunidad Valenciana. Valencia: Instituto Valenciano de Estadística.

Choay, F. (1994). Nueva Babel. El reino de lo urbano y la muerte de la ciudad. Revista Arquitectura Viva, No. 35, pp. 15-22.

Downing, P.B. (1977). Local service pricing policies and their effect on urban spatial structure. Vancouver: University of British Columbia Press.

Ewing, R.; Schmid, T.; Killingsworth, R.; Zlot, A. \& Raudenbush, S. (2008). Relationship between urban sprawl and phisical activity, obesity and morbidity. En J.M. Marzlutt et al. (Eds.). Urban Ecology. An international perspective on the interaction between human and Nature. New York: Springer.

Generalitat Valenciana (2001). El transporte metropolitano del área de Alicante. Encuesta domiciliaria de movilidad. Velencia: Generalitat Valenciana.

Gillham, O. (2002). The limitless city: a primer on the urban sprawl debate. Washington D.C.: Island Press.

Hall, P. (1995). A European perspective on the spatial links between land use, development and transport. En D. Banister, (Ed.). Transport and urban development. Londres: Spon.

Herce, M. \& Magriñá, J. (2002). El soporte infraestructural de la ciudad. Barcelona. Ediciones UPC. 
Indovina, F. (1990). Citta difusa. Venecia: Daest-IUAV.

Jacobs, J. (1971). La economia de las ciudades. Madrid: Ed. Península.

Jacobs, J. (1973). Muerte y vida de las grandes ciudades. Madrid: Ed. Península.

Kaido, K. (2006). Urban densities, quality of life and local facility accessibility in principal Japanese cities. En M. Jenks \& N. Dempsey (Eds.). Future forms and design for sustainable cities. Oxford; Burlington, MA: Architectural Press.

Lynch, K. (1961). The Image of the City. Cambridge, MA: The MIT Press.

Lynch, K. (1981). A theory of Good City Form? Barcelona: Gustavo Gili.

Lynch, K. \& Rodwin, L. (1958). A theory of urban form. Journal of the American Institute of Planners, No. 4.

La Caixa. Servicio de Estudios (2009). Anuario Económico de España. Barcelona: La Caixa.

Lefevbre, H. (1972). La Revolución Urbana. Madrid: Alianza Editorial. 1972.

Lever, W.F. (1962). Planning Standard and residential densities. Journal of the Town Planning Institute, Vol. 57, No. 9.

López de Lucio, R. (2007). Construir ciudad en la periferia. Madrid: Mairea Libros.

March, L. (1975). Modelos elementales de formas de la edificación. En L. Martín (Ed.). La Estructura del Espacio Urbano. Barcelona: Gustavo Gili.

Martín, L. (Ed.). La Estructura del Espacio Urbano. Barcelona: Gustavo Gili.

Meadows, H.D. (1972). Los limites del crecimiento. Informe del Club de Roma sobre el predicamento de la humanidad. México D.F.: Fondo de Cultura Económica.

Mumford, L. (1966). La Ciudad en la Historia. Buenos Aires: Infinito.

Navarro, J.R. \& Ortuño, A. (No publicado). Estudio sobre el impacto territorial de los campos de golfy operaciones asociadas en el Levante español. Madrid: Ministerio de Medio Ambiente.

Newman, P. \& Kenworthy, J. (1999). Sustainability and cities: overcoming automobile dependence. Washington D.C.: Island Press.

Richardson, H. \& Gordon, P. (1997). Are compact cities a desirable planning goal? Journal of the American Planning Association, Vol. 63, No. 1, pp. 95-107.

Samoná, G. (1975). La cittá in stensione. Revista Espacio y Sociedad, No. 2.

Solá-Morales, M. (1997). Territoris sense model. Papers, No. 26.

Soria, A. (1979). Ildefonso Cerdá. Hacia una Teoría General de la Urbanización. Madrid: Ed.Turner y Colegio de Ingenieros de Caminos.

Wheaton, W.L. \& Schussheim, M. J. (1955). The cost of municipal services in residential areas. Washington D.C.: Dept.of Commerce.

Weber, M. (1970). Indagaciones sobre la estructura urbana. Barcelona: Gusttavo Gili. 